Ann. Zootech., I977, 26 (3), 42I-428.

\title{
Note
}

\section{Effet de l'addition de méthionine au régime sur les caractéristiques du comportement alimentaire du lapin en croissance}

\author{
M. PRUD'HON, M. COLIN * et F. LEBAS * \\ avec la collaboration technique de J. Angevain et J. Goussopoulos \\ Station de Physiologic animale, I.N.R.A.. \\ E.N.S.A., Place Viala, 34060 Montpellier Cedex (France). \\ * Station de Recherches sur l'Elevage du Lapin. \\ Centre national de Recherches zootechniques, I.N.R.A. \\ 78350 Jouy-en-Josas (France)
}

\begin{abstract}
Résumé
Vingt quatre lapins Néo-Zélandais blancs, âgés de 5 semaines, répartis en deux groupes, ont été placés pendant 4 semaines dans un dispositif d'enregistrement graphique des consommations alimentaires individuelles. La moitié d'entre eux a reçu un aliment partiellement carencé en acides aminés soufrés $(0,43$ p. Ioo) tandis que l'autre moitié recevait le même aliment supplémenté par 0,2 p. Ioo de DL-méthionine; après deux semaines, les régimes ont été inversés. Au cours de chaque période, les lapereaux recevant le régime supplémenté ont eu une vitesse de croissance plus rapide et un indice de consommation diminué. Aucune différence significative n'est apparue dans la fréquence des repas solides, dans les quantités d'aliment consommées ni dans la répartition de ces consommations au cours du nycthémère. Ces résultats sont en contradiction, sur certains points, avec ceux obtenus précédemment à partir des régimes de même composition; les causes possibles sont passées en revue.
\end{abstract}

\section{Introduction}

Des travaux récents ont montré que la méthionine est un acide aminé indispensable au lapin en croissance (ADAMSON et FISHER, I97I). Le niveau des besoins paraît varier selon les régimes étudiés (CheEke, I97 I; Colin, ARKHuRst et Lebas, I973; Colin, I975) notamment en fonction de la concentration en énergie digestible des aliments. Ces auteurs ont montré que l'insuffisance ou l'excès d'acides aminés soufrés dans la ration s'accompagne d'une réduction des consommations spontanées qui cesse dès que le régime est rééquilibré. Qu'il s'agisse de méthionine ou d'un autre acide aminé, la supplémentation par l'acide aminé limitant entraîne une modification rapide et de grande amplitude de la quantité d'aliment ingéré (CoLIN, 
Lebas et Delavead, I975, Hamidou, I975, Colin, I976). Aucun expérimentateur n'a cherché à comparer systématiquement les caractéristiques des consommations solides et /ou liquides de lapins recevant un régime équilibré ou un régime déficient en acides aminés soufrés. Nous avons donc tenté de préciser chez le Lapin, l'influence de la supplémentation en méthionine d'une ration partiellement carencée, sur certains paramètres du comportement alimentaire. Cette étude a été nise en place dans les installations de contrôle du comportement alimentaire du Lapin, à l'E.N.S.A. de Montpellier (PRUD'Hon et al., I972).

\section{Matériel et méthodes}

\section{Animaux}

Vingt-quatre lapins des deux sexes, de race "Néo-Zélandais blanc ", ont été répartis en deux lots. Ils étaient âgés de $37 \pm 2$ jours au début des contrôles. Ils ont été placés en cage d'enregistrement individuel en quatre séries de six animaux. entre le mois d'octobre et de février 1976; chaque période de contrôle a duré quatre semaines. Pendant les deux premières semaines la moitié des lapereaux a reçu le régime $A$, l'autre le régime $B$; au cours des deux semaines suivantes les régimes ont été inversés, ceci afin de pouvoir enregistrer l'effet immédiat d'un changement de régime sur les composantes du comportement alimentaire, chaque lapin étant son propre témoin.

\section{Régimes expérimentaux}

Les régimes utilisés étaient principalement à base de luzerne déshydratée, de tourteau de soja et de tournesol et d'amidon de maïs (tabl. I), la méthionine supplémentaire (o, 2 p. Ioo du régime) remplaçant le glutamate de sodium du régime de base en quantité équivalente.

TABLEAU I

Composition des régimes expérimentanx

Composition of the experimental diets

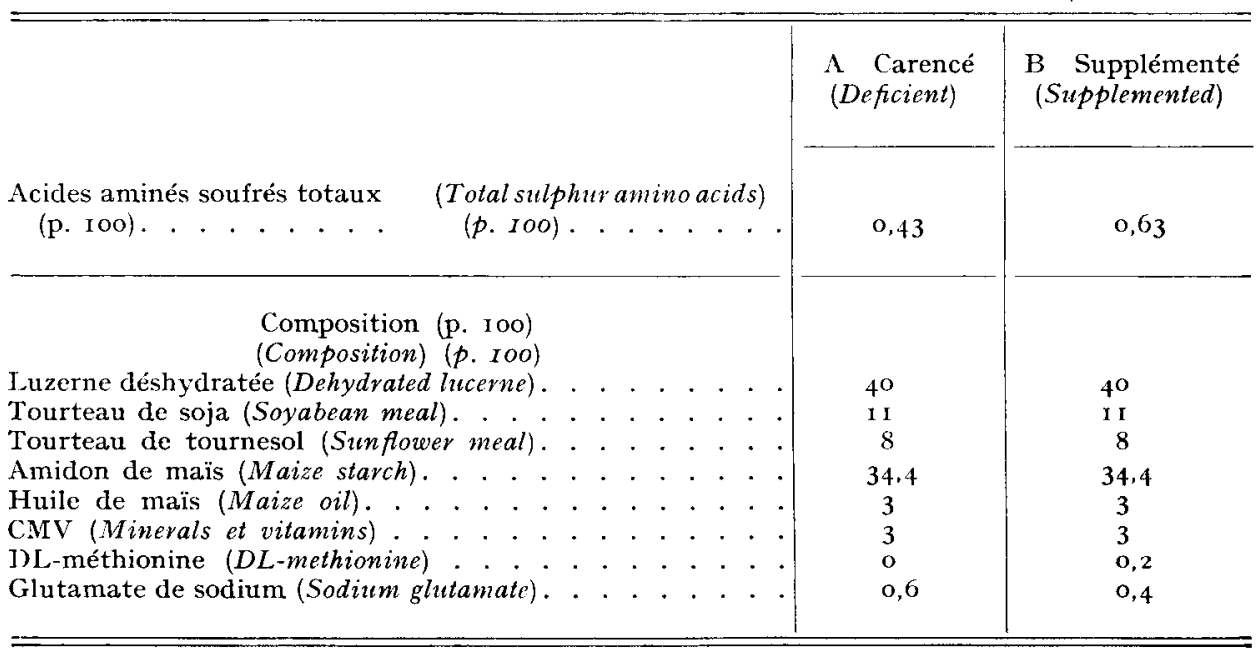


Les rations apportaient $2660 \mathrm{Kcal} / \mathrm{kg}$ d'énergie digestible, I6 p. Ioo d'azote, $0,7 \mathrm{I}$ p. Ioo de lysine et respectivement 0,43 et 0,63 p. Ioo d'acides aminés soufrés. Ces deux régimes avaient déjà été utilisés dans des travaux précédents par l'un d'entre nous (Colin, I975).

Les aliments étaient présentés à volonté, sous forme de granulés de $2,5 \mathrm{~mm}$ de diamètre.

\section{Contrôles sur les animaux}

Les animaux ont été placés dans les cages d'enregistrement continu des consommations d'aliments solides et liquides, décrites précédemment (PRUD'HON et al., I972, PRUD'HON, I973) dans un local maintenu à $20 \pm \mathrm{I}^{\circ} \mathrm{C}$, éclairé de 7 heu-

TABLEAU 2

Principaux résultats

Main results

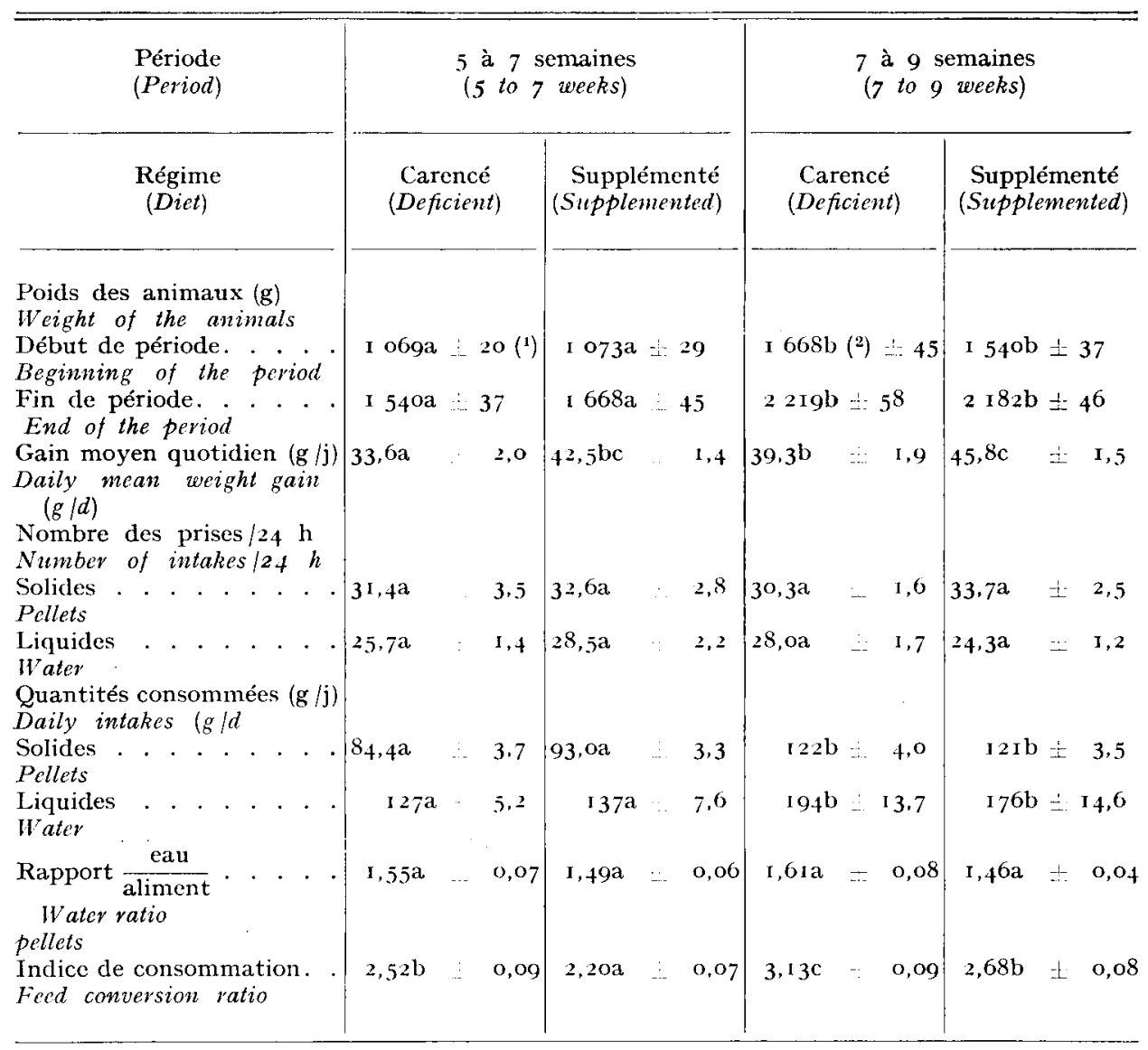

(1) Moyenne

$\left({ }^{2}\right)$ Sur une même ligne, les valeurs ayant une lettre différente en indice different significativement au seuil $\mathrm{P}=0,05$. (On the same line values with the same letter are not statistically different at the level $P=$ 0.05$. 

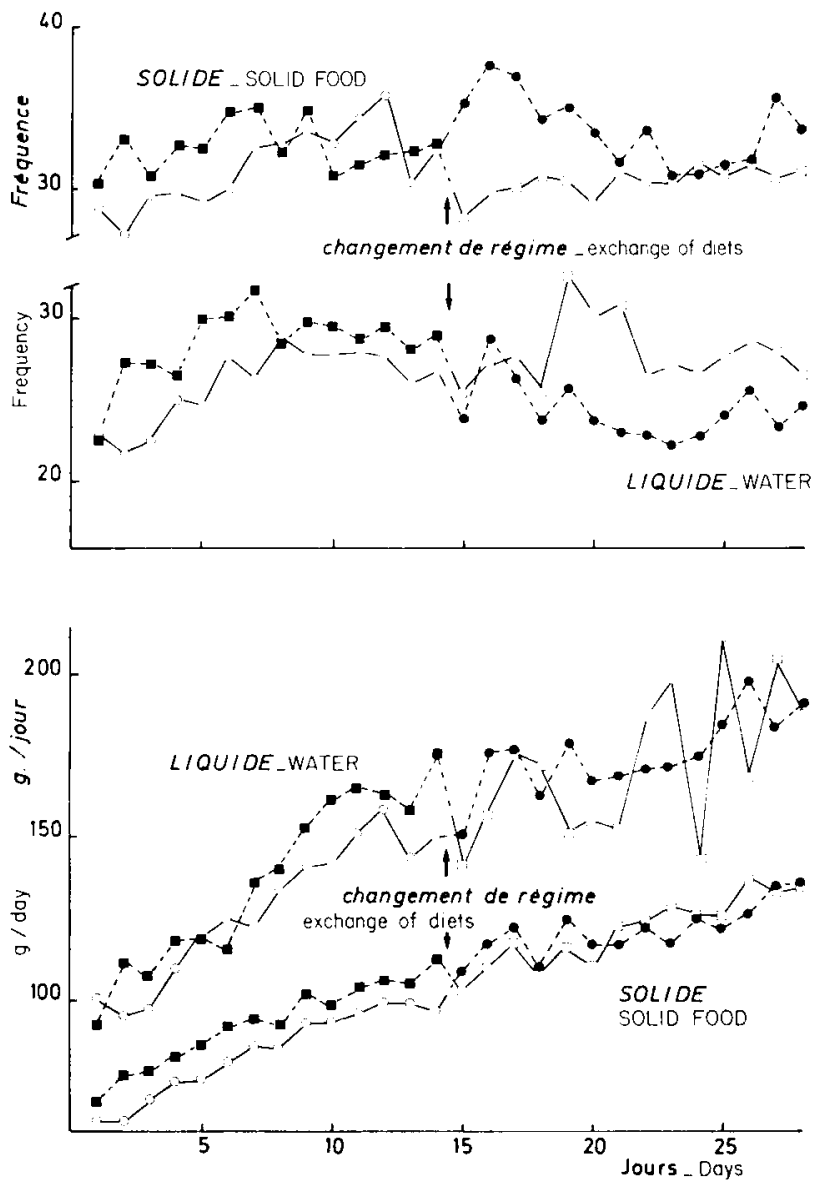

FIg. I. - Évolution au cours de la durée totale de l'essai, de la fréquence des prises d'aliments solide et liquide et des quantitées consommées chaque jour.

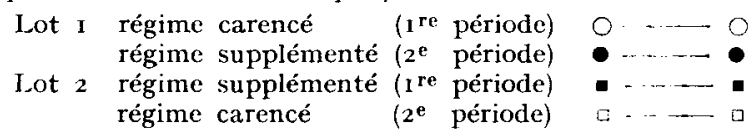

Frequency of feed and water intakes and daily feed intake level during the wole cxperiment.

\begin{tabular}{|c|c|c|c|c|c|}
\hline Group & I & deficient diet & (Ist & period) & \\
\hline & & supplemented diet & (2nd & period) & — \\
\hline & 2 & $\begin{array}{l}\text { supplemented diet } \\
\text { deficient diet }\end{array}$ & $\begin{array}{l}\text { (Ist } \\
\text { (2nd }\end{array}$ & $\begin{array}{l}\text { period) } \\
\text { period) }\end{array}$ & 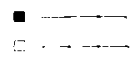 \\
\hline
\end{tabular}


res à 2 I heures. Le moment exact et l'amplitude de chaque prise effective d'aliment solide et d'eau de boisson ont été enregistrés.

Les lapins ont été pesés une fois par semaine à 8 h 30 , après la période de forte consommation nocturne.

\section{Exploitation des résultats}

Les paramètres mesurés ont été analysés par jour et chaque jour par heure du nycthémère, des études précédentes nous ayant montré que les modifications de comportement alimentaire affectent essentiellement la période de consommation comprise entre I5 et 2I heures (PRUD'HON et al., I975, PRUD'HON, I976). Les effets du régime sur les différents paramètres étudiés ont été comparés à l'aide du test «t).

\section{Résultats}

La supplémentation du régime de base avec 0,2 p. Ioo de DL méthionine a entraîné un accroissement significatif de la vitesse de croissance et un abaissement de l'indice de consommation (tabl. 2).

Pour tous les autres paramètres étudiés - fréquence des prises solides et liquides, quantités consommées, rapport eau /aliment - nous n'avons pas enregistré d'effet significatif d'une supplémentation en acides aminés soufrés; toutefois, au cours de la période 5 à 7 semaines, les lapins recevant l'aliment supplémenté ont consommé régulièrement une quantité de granulés plus élevée que celle ingérée par les lapins alimentés avec l'aliment carencé; cependant la différence n'est pas significative. Les lapins ayant eu un aliment pauvre en acides aminés soufrés entre 5 et 7 semaines ont compensé leur retard de croissance entre 7 et 9 semaines lorsqu'ils disposaient d'un aliment équilibré.

Le passage d'un régime à l'autre n'a pas eu d'incidence nette sur la fréquence des prises ni sur le niveau de consommation des aliments granulés et de l'eau de boisson (fig. I).

La répartition des consommations solides et liquides au cours du nycthémère a été rigoureusement semblable pour les deux régimes et les deux périodes étudiées (fig. 2).

\section{Discussion et conclusion}

Comme dans nos essais précédents (Colis, r975), l'addition de o,z p. roo de méthionine à un régime contenant $0,43 \mathrm{p}$. Ioo d'acides aminés soufrés améliore très sensiblement la vitesse de croissance des lapins $(+26$ p. Ioo entre 5 et 7 semaines, + I 7 p. Ioo entre 7 et 9 semaines). Contrairement aux observations précédentes (Arkhurst, I973, Colin, I975), l'amélioration des performances est due essentiellement à une efficacité accrue de l'utilisation de l'aliment sans modification sensible du niveau d'ingestion alimentaire. Dans plusieurs essais de supplémentation des régimes en DL méthionine nous avions déjà constaté une amélioration non significative des indices de consommation lorsque la vitesse de croissance est maximale. Ces essais ayant été réalisés avec des lapins "Néo-Zélandais ", et non des "Californiens " ou des " croisés ", comme précédemment, on peut penser à un origine génétique de la différence de réponse à la supplémentation. Par ailleurs, les croissances rapides relevées dans les deux lots entre 7 et 9 semaines permettent de penser 

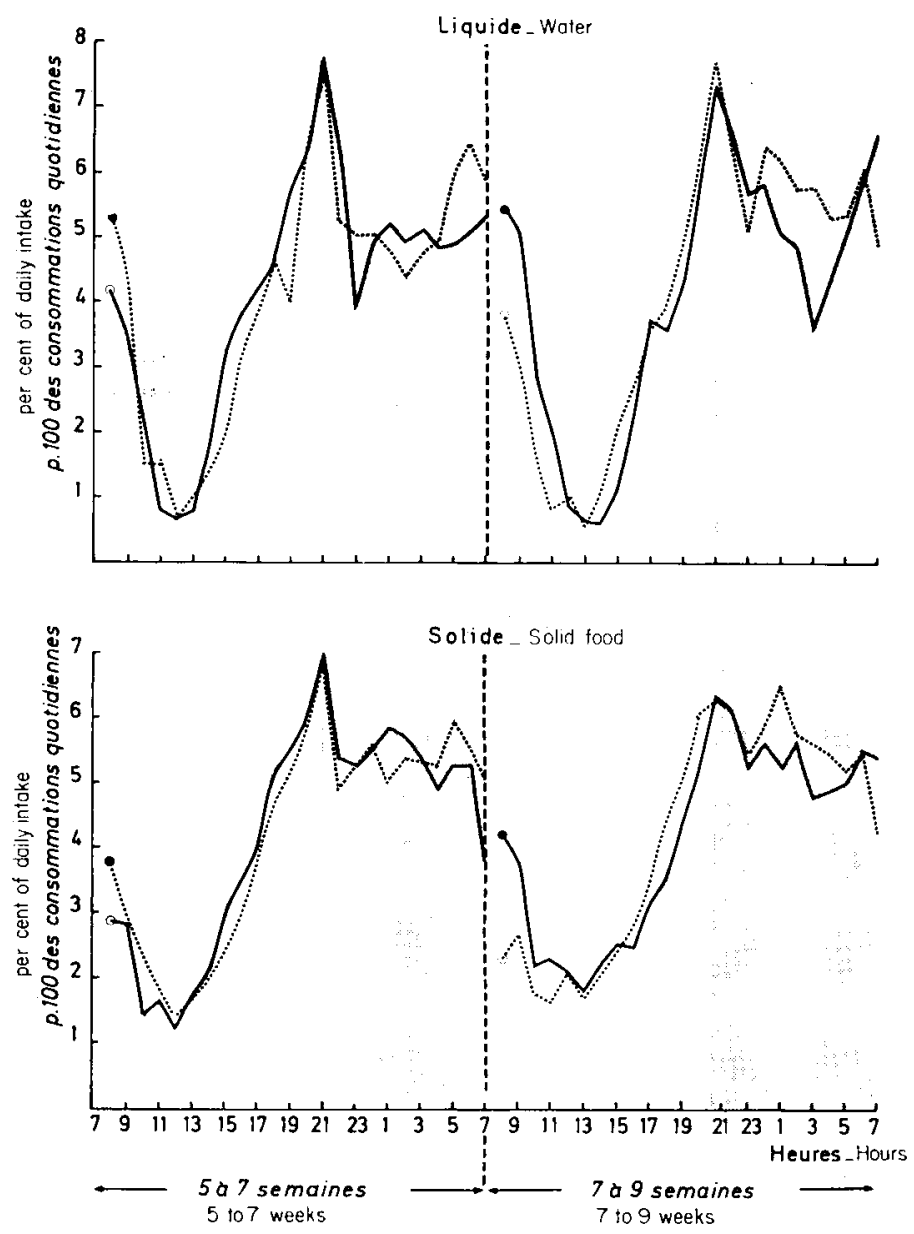

FIG. 2. - Répartition an cours du nycthémère des consommations horaires d'aliments solideet liquide exprimées en pourcentage de la consommation totale quotidienne, moyenne pour chacune des 2 périodes expérimentales.
Lot I régime carencé (I re période) régime supplémenté ( 2 période)
Lot 2 régime supplémenté ( $1^{\text {re }}$ période) régime carencé (2 ${ }^{\mathrm{e}}$ période)

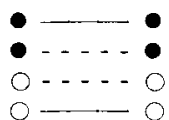

Distribution during the 2 thr cycle of the hourly feed and water intakes, as a per cent of total mean daily intakes, for each of the 2 experimental periods.

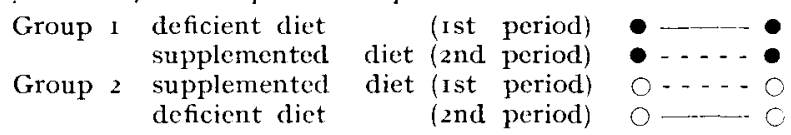

qu'à ce stade les besoins en acides aminés soufrés sont pratiquement couverts par le régime non supplémenté. Il est probable que chez le Lapin comme chez le Porc (FAUCONNEAL et Pion, I972) le besoin en acides aminés soufrés, exprimé en pourcentage du régime, diminue avec l'âge.

L'absence de modifications sensibles du niveau d'ingestion des aliments ex- 
plique sans doute l'absence complète de modifications des diverses caractéristiques du comportement alimentaire et suggère la nécessité de poursuivre cette étude en utilisant des régimes plus déséquilibrés en acides aminés soufrés.

En conclusion, cet essai confirme l'intérêt réel d'une supplémentation en méthionine amenant la teneur de la ration au voisinage de 0,60 p. Ioo. Il montre également que le besoin en acides aminés soufrés du Lapin varie vraisemblablement avec certaines caractéristiques des animaux, notamment leur âge. Mais ce dernier point devra faire l'objet d'études complémentaires.

Accepté pour publication en avril 1977.

\section{Summary}

\section{Effects of methionine supplementation on feeding behaviour characteristics of growing rabbits}

Twenty four white New-Zealand rabbits, aged 5 weeks, were allocated into two groups and placed during 4 weeks in an individual feed intake recording device. One group was fed a diet poor in sulphur amino acids $(0.43 \%)$, the other group was fed the same ration but supplemented with 0.2 per cent DL-methionine (Table I). After two weeks, the treatments were inversed. During each period, the rabbits receiving the methionine supplemented diet showed a higher growth rate and lower feed conversion ratio (Table 2). No significant difference was observed either in the frequency of solid meal intake, or the intake level or the feeding pattern during the 24 hr-cycle. These results are in contradiction, on some aspects, with those obtained previously with similar diets; the possible causes are discussed.

\section{Références bibliographiques}

Adamson I., Fisher H., I97I. The amino acid requirements of the growing rabbit: qualitative needs. Nutr. Rep. Int., 4, 59-64.

Arkhurst G., 1973. Effet chez le Lapin en croissance de l'addition de DI-méthionine au régime alimentaire. Thèse Doct. $3^{\mathrm{e}}$ cycle. Univ. Paris-Vle.

Chee ke P. R., I97I. Arginine, lysine and methionine needs of the growing rabbit. Nutr. Rep. Int., 5, 259-266.

Courn M., 1975. Effets sur la croissance du Lapin de la supplémentation en L-lysine et en DLméthionine de régimes végétaux simplifiés. Ann. Zootech., 24, 465-474.

Colın M., r976. Étude des possibilités de supplémentation de régimes carencés par apport de lysine ou de méthionine dans l'eau de boisson chez le Lapin. I er Congr. int. Cunic. Dijon, 18, I-4.

Colin M., Arkhurst G., Lebas F., i973. Effets de l'addition de méthionine au régime alimentaire sur les performances de croissance chez le Lapin. Ann. Zootech., 22, 485-491.

Colin M., Lebas F., Delaveau A., 1975. L'influence d'un apport de lysine dans l'aliment solide ou dans l'eau de boisson sur les performances de croissance du Lapin. Ann. Zootech., 24, 3 II $-3 I 3$.

Fauconneau G., Pion R., I972. Quelques aspects nouveaux du métabolisme azoté chez le Porc et leurs conséquences pour la satisfaction de ses besoins en protéines. Ann. Zootech., 21, $275^{-297 .}$

Hamidou O., r975. Étude du taux optimum d'acides aminés soufrés dans l'alimentation du lapin. Comparaison de deux modalités d'apport de DL-méthionine supplémentaire. Diplôme Études approfondies Université Paris VI.

PRUD'HoN M., I973. Le comportement alimentaire du Lapin en croissance. Mise au point d'une méthode d'étude et premières observations. Congr. Int. Cóme. Sept. 73, 3I7-325. 
Prud'hon M., 1976. Comportement alimentaire du lapin soumis aux températures de Io, 20 et $30^{\circ} \mathrm{C}$. I er Congr. int. Cunic., Dijon, 14, I-6.

Prud'hon M., Carles Y., Goussopoulos J., Koehl P. F., I972. Enregistrement graphique des consommations d'aliments solide et liquide du Lapin domestique nourri ad libitum. Ann. Zootech., 21, 45I-460.

Prudinon M., Cherubin M., Goussopoulos J., Carles Y., 1975. Évolution au cours de la croissance des caractéristiques de la consommation d'aliments solide et liquide du I apin domestique nourri ad libitum. Amn. Zootech., 24, 289-298. 